\title{
REDIRECTED SCENE ROTATION FOR IMMERSIVE MOVIE EXPERIENCES
}

\author{
An Undergraduate Research Scholars Thesis \\ by \\ TRAVIS STEBBINS \\ Submitted to the Undergraduate Research Scholars program at \\ Texas A\&M University \\ in partial fulfillment of the requirements for the designation as an \\ UNDERGRADUATE RESEARCH SCHOLAR
}

Approved by Research Advisor:

Dr. Eric Ragan

May 2018

Major: Visualization

Computer Science 


\section{TABLE OF CONTENTS}

Page

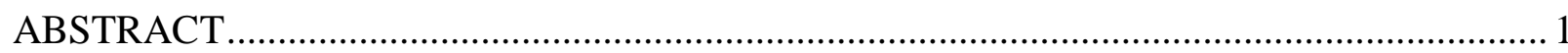

ACKNOWLEDGMENTS ........................................................................................ 2

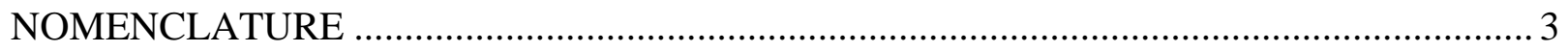

\section{CHAPTER}

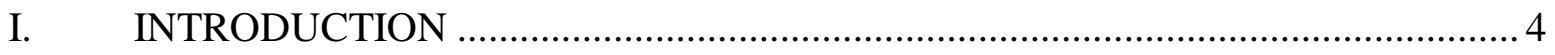

II. REDIRECTION FOR IMMERSIVE MOVIES ............................................ 6

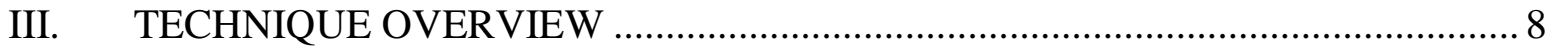

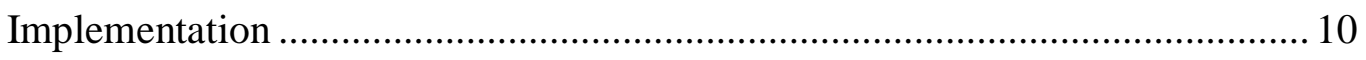

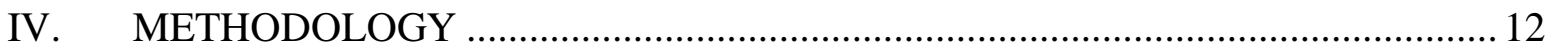

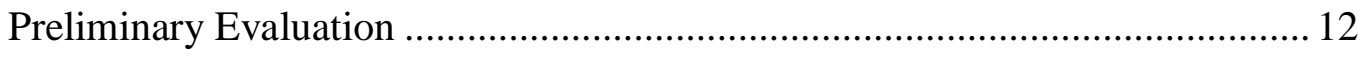

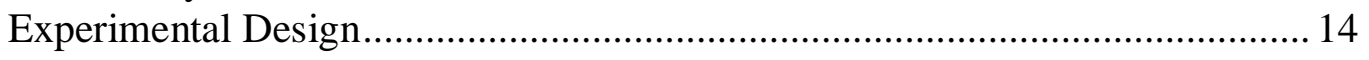

Testing Environment and Parameters …................................................... 14

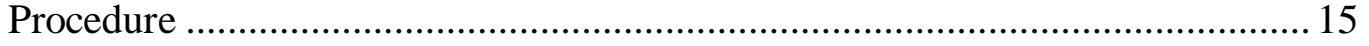

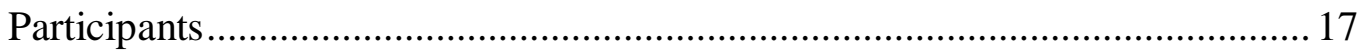

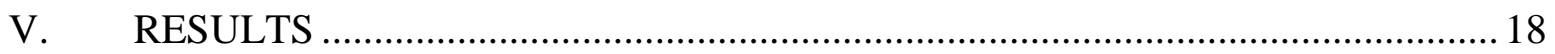

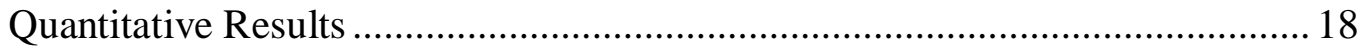

Qualitative Results .............................................................................. 23

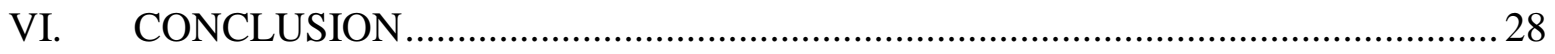

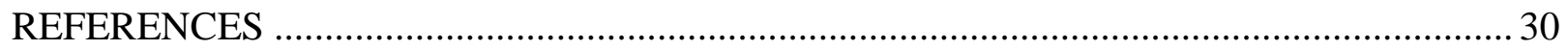

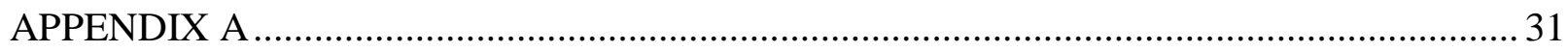

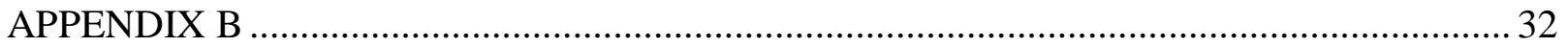




\author{
ABSTRACT \\ Redirected Scene Rotation for Immersive Movie Experiences \\ Travis Stebbins \\ Department of Visualization \\ Texas A\&M University
Research Advisor: Dr. Eric Ragan
Department of Visualization
Texas A\&M University

Virtual reality (VR) allows for immersive and natural viewing experiences; however, these often expect users to be standing and able to physically turn and move easily. Seated VR applications, specifically immersive 360-degree movies, must be appropriately designed to facilitate user comfort and prevent sickness. Our research explores a scene rotation-based method for redirecting a viewer's gaze and its effectiveness given two parameter adjustments: rotation speed and delay/angle threshold. The research explores the feasibility and effectiveness of the technique and of variations of the parameter values. The research is important because the results will prove useful in the development of future immersive movie or virtual reality experiences. We conducted a controlled user study to determine how users responded to the scene rotation and which parameter values they preferred. Metrics for effective results are derived from user comfort, sickness, and overall preference. From our study, we discovered that users responded favorably to the scene rotation technique, especially for the slow rotation speed. The results of this research will further the understanding of how to effectively develop content for virtual reality systems. 


\section{ACKNOWLEDGEMENTS}

I would like to thank my research advisor, Dr. Eric D. Ragan, for his assistance in conducting this research and his guidance and mentorship throughout the course of this research.

Furthermore, I would like to thank the Department of Visualization at Texas A\&M University for providing me with the skills and resources necessary to effectively conduct the studies for this research.

Finally, I would like to thank my friends and family for their support, assistance, and feedback throughout the research process. 


\section{NOMENCLATURE}

$\begin{array}{ll}\text { VR } & \text { Virtual Reality } \\ \text { VE } & \text { Virtual Environment } \\ \text { HMD } & \text { Head-mounted display } \\ \text { D/AT } & \text { Delay/Angle Threshold }\end{array}$




\section{CHAPTER I}

\section{INTRODUCTION}

Virtual reality (VR) systems bring a range of benefits for experiencing 3D simulated worlds [3]. The use of advanced display and interaction features allows users to experience detailed, engaging, and interactive narratives for entertainment. Recently, many commercial VR experiences have been presented as forms of immersive stories or movies that bring new opportunities for story telling through a highly interactive medium. For such applications, interactive view control is assumed as a requirement to be considered a VR experience, especially since head-tracking capabilities are supported as core "immersive" elements for headworn systems such as the Google Daydream, Oculus Rift, or HTC Vive.

However, while many designers often assume the use of VR where users stand and are free to physically turn without constraints, this excludes many commonly desired usage settings where the user is seated [6], such as use of VR while relaxing on the couch or passing the time on a plane during a flight. For such situations, it may be uncomfortable to have the head physically turned for much time. To address this problem, we study the use of a redirected scene rotation technique to allow the viewer's gaze to gradually redirect towards the straight-ahead physical direction during immersive movie experiences. We employ a method similar to washout filters used for motion simulators, which can be used to gradually "pull the position of the simulator back to its neutral position" over time [4]. By rotating back to the neutral orientation, the HMD is able to simulate a greater range of motion than would be physically comfortable in seated VR (or more than would be physically possible in the case of motion simulation). These 
washout filters aim to reorient the user without the user noticing the motion through their visual or vestibular senses [4].

In this research, we explore the use of washout filters as a form of redirection that can be applied to VR experiences where limited physical turning is possible. We aim to study variations of the washout method with different degrees of rotation speed and delay/angle thresholds to reorient the physical head orientation while users watch immersive movies. In our poster and extended abstract [8] for the IEEE VR 2018 conference, we presented an early design along with a preliminary evaluation and results. In this paper, we expand on our research and conduct controlled user studies focusing on user comfort, immersion, sickness, and overall preference. We present our technique design and controlled study results to determine the effectiveness of the technique and the influence of the parameter values on an immersive movie experience. 


\section{CHAPTER II}

\section{REDIRECTION FOR IMMERSIVE MOVIES}

An important aspect to consider when developing any VR technique is motion sickness, which has proven challenging when developing motion-based VR experiences [1]. One promising technique for overcoming some of the limitations with VR is redirection. Many researchers have studied the use of redirection techniques for gradually adjusting viewer gaze in interactive VR experiences (e.g., [2, 5, 7]). While redirected walking techniques primarily deal with redirecting and reorienting a user that is physically moving or walking through space to navigate $[4,6]$, researchers have also explored gradual redirection in stationary experiences. For example, Sargunam et al. [6] presented a guided rotation technique for seated VR that is applied dynamically as the user virtually travels through the 3D world; however, this technique had limitations for free navigation in 3D spaces due to the need for slow view adjustments. In our research, we focus on experiences in which the user has head-tracked view control but does not have interactive control of translational movement. In particular, we study a variation of redirection applied to viewing immersive movies.

This technique may be effective for immersive narrative or cinematic experiences that have a central focal point in the experience - that is, a direction where the designer or director expects the user to look for the majority of time. For such applications, even if this focal point moves in such a way that requires scene rotation to follow the target, the viewer will often have limited need to freely look around for an extended amount of time. As a result, the focal point can more easily be kept in alignment with the physical forward direction to reduce physical discomfort associated with extreme or awkward head or body rotations during seated viewing. 
Schmitz et al. [7] provide further evidence that a scene redirection technique for immersive movies may be viable. In their paper, they discuss the "Threshold of Limited Immersion," which is their proposed measure for detecting when a user's "immersion breaks in a real application scenario" [7]. Their research demonstrates that traditional detection thresholds are often too conservative for interactive experiences that demand a user's attention. Indeed, they found that they were able to apply rotation gains significantly greater than traditional detection thresholds without breaking the user's immersion. While their research covers redirected walking techniques and rotational gain, the same concept may apply to immersive movies in VR, which also frequently demand a user's attention and provide a situation that may distract the user from scene rotation. 


\section{CHAPTER III}

\section{TECHNIQUE OVERVIEW}

Our redirected scene rotation technique uses an approach similar to that found in motion simulator washout filters but for VR movies with head-worn displays. The technique assumes a default head orientation, which is assumed to be a comfortable viewing position where the user is looking straight ahead. We call this the neutral position. The technique is designed to reduce the amount of time spent turning away from the neutral position based on the premise that too much time spent turning towards the side can cause physical discomfort. Thus, our technique is initiated after the user rotates their head to an extreme enough angle and remains for a given amount of time. After turning to the side, the technique will slowly rotate the virtual scene such that the viewer can will be able to maintain focus on the same point of interest while that focal point is brought back to align with the neutral physical position. The technique accomplishes this
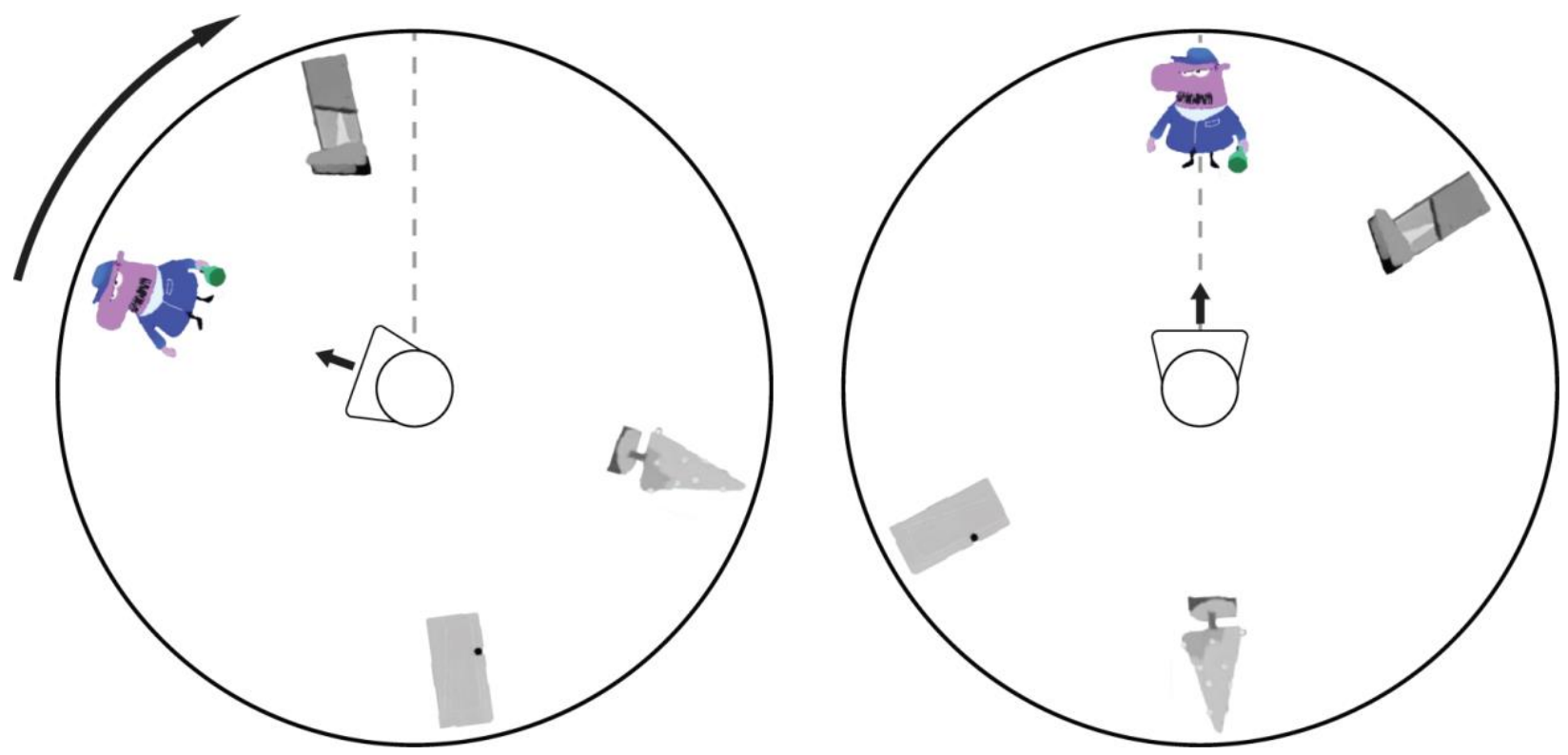

Figure 1. Example Scene Rotation. The redirected scene rotation adjusts the virtual viewing orientation while the user follows the focal point (in this case, the colorful purple and blue character). 
by slowly rotating the entire scene of the immersive movie, causing the user's focus to be shifted directly in front of them; this essentially allows the focal point of the immersive movie to always be placed toward the front of the user's body so that it can be viewed in a comfortable position.

If the focal point again moves to one side of the user, the same process can be repeated. Figure 1 shows an example of how the process works and how the scene is rotated to shift the focal point.

To reduce any visual-vestibular discrepancies as much as possible, a Bézier curve with flat tangents at the endpoints is used to interpolate the rotation of the scene; this ensures a smooth acceleration. Given the angle of the user's gaze, a corresponding scene rotation is calculated that will rotate the scene to such a position that the user's head returns to a neutral orientation. In our implementation, the magnitude of this rotation angle determines the duration of time over which the scene will rotate. There is a direct relationship between rotation angle magnitude and rotation duration; this ensures that the scene always rotates with the same average speed, as specified in the filter parameters. The scene then rotates from the initial angle to the

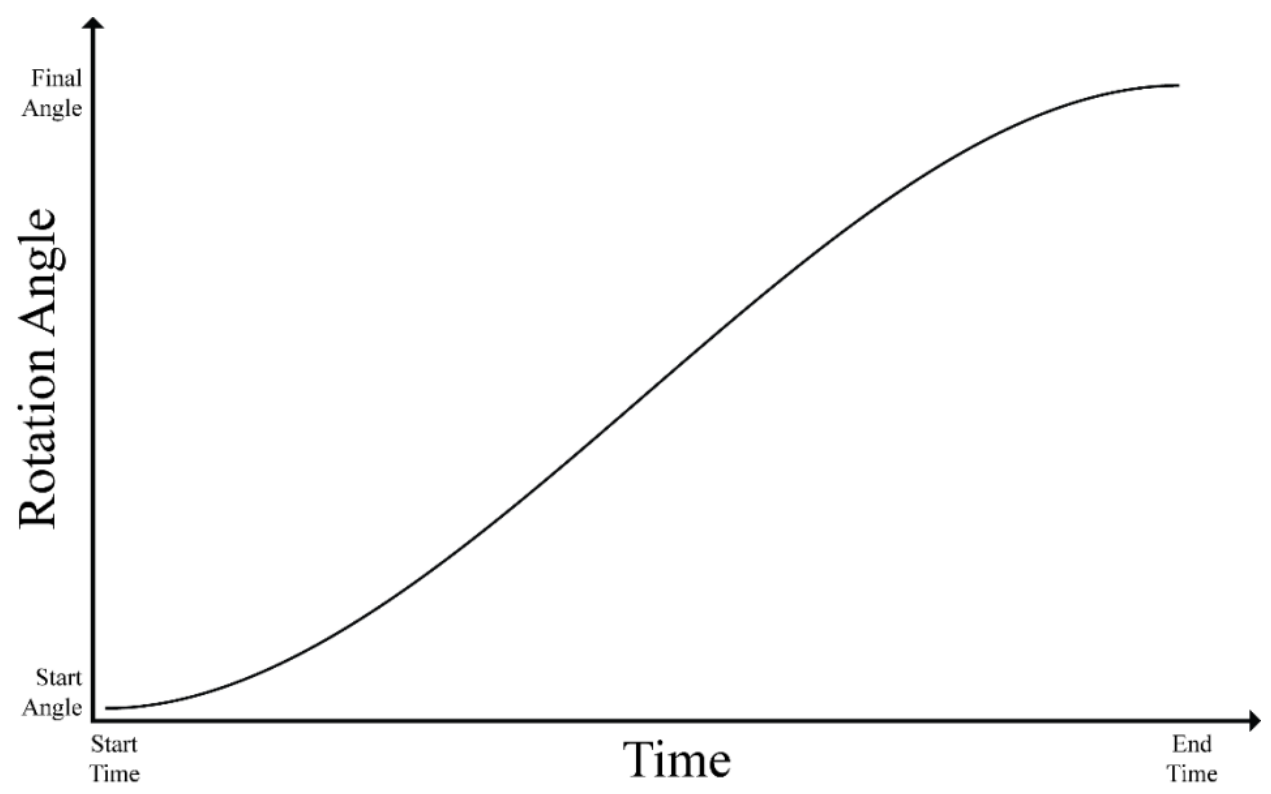

Figure 2. Example scene rotation curve. The Start Angle corresponds to the scene's initial rotation, and the Final Angle corresponds to the angle that will return the user's head to the straight ahead physical direction. The duration between the Start Time and End Time has a direct relationship with the magnitude of the rotation angle, so as to keep a constant average rotation speed. 
$x=(1-t)\left((1-t)\left((1-t) x_{0}+t x_{1}\right)+t\left((1-t) x_{1}+t x_{2}\right)\right)+t\left((1-t)\left((1-t) x_{1}+t x_{2}\right)+t\left((1-t) x_{2}+t x_{3}\right)\right)$

$y=(1-t)\left((1-t)\left((1-t) x_{0}+t x_{1}\right)+t\left((1-t) x_{1}+t x_{2}\right)\right)+t\left((1-t)\left((1-t) x_{1}+t x_{2}\right)+t\left((1-t) x_{2}+t x_{3}\right)\right)$

where $\quad 0 \leq t \leq 1$,

$x_{0}=$ start time, $x_{1}=x_{2}=$ halfway time, $x_{3}=$ end time,

$y_{0}=y_{1}=$ start angle, $y_{2}=y_{3}=$ final angle

Figure 3: Parametric equation for the Bézier curve used for scene rotation.

calculated angle following the smooth Bézier curve to transition the orientation to the intended rotation. Figure 2 shows an example of the curve used for scene rotation. Figure 3 gives the parametric equation for the Bézier curve.

Furthermore, a rotation override is implemented in the technique such that if the user rotates his or her head faster than a specified speed while the scene is rotating, the scene rotation is stopped. We discovered the need for this override after our preliminary evaluation, in which some users complained that the scene would continue to rotate after they had stopped moving their head or while they were trying to shift their gaze to look at another point in the scene. By using this scene rotation override, we reduce the likelihood of any unnecessary or undesirable scene rotation from occurring.

\section{Implementation}

We developed a working implementation of the redirected scene rotation technique in a custom video movie player using the Unity game engine (version 2017.3.1f). For the implementation, immersive movies are displayed on an inverted sphere that surrounds the user, and we created scripts to implement the technique. The movie player tracks where a user has been looking and for how long; if the user has looked at an extreme enough angle (we call this angle the angle threshold) for a long enough time (we call this time the rotation delay), then the scene rotation is triggered. The appropriate scene rotation angle is calculated and the scene then rotates at a specified rotation speed (which can be parameterized according to preference or requirements for a specific video). This scene rotation rotates the scene in the direction of the desired physical rotation, which in turn encourages the user to physically turn with the scene to 
return to the neutral viewing orientation. Additionally, if the user turns his or her head fast enough during the scene rotation, then the override is triggered, and the scene rotation stops.

Because the research is focused solely on viewing immersive movies in VR, only physical head rotation is mapped to virtual head rotation in the technique implementation; head translation is not implemented, as we deemed it unnecessary for testing of the technique. Users have full 6 degrees of freedom of rotation for head tracking.

Additionally, for use in data analysis, our implementation of the technique records the user's head rotation at each frame of the video and outputs the current time and head rotation angles $(\mathrm{x}, \mathrm{y}$, and $\mathrm{z})$ to a text file. 


\section{CHAPTER IV}

\section{METHODOLOGY}

We conducted a controlled study with a primary focus on testing parameters for the redirected scene rotation technique. The main goals of our study were: (1) to determine the effects of rotation speed and delay/angle threshold ratio on user experience, and (2) to gauge overall user preference for the parameter values and scene rotation in general.

\section{Preliminary Evaluation}

Before running the controlled study, we first conducted a conducted a smaller preliminary evaluation to help refine the technique and study methodology. The preliminary evaluation of the technique used three immersive movies from 360 Google Spotlight Story: Rain or Shine, Special Delivery, and Buggy Night. These are all short, good-natured, digitally animated narrative videos that last between approximately 3 and 5.5 minutes. For example, Rain or Shine is about an unlucky girl whose sunglasses cause a rain cloud to form above her, bringing a constant torrential downpour. Special Delivery is about a grumpy animated character attempting to catch Santa Claus. Buggy Night is about a group of small bugs attempting to escape being eaten by a frog. Figures 4-6 show screenshots from each of the videos. The preliminary evaluation was conducted using a Samsung Galaxy S7 edge and a Samsung Gear VR system. We ran the preliminary evaluation with 5 participants. In each trial, the user viewed one or two of the immersive movies one to three times with different parameters during each viewing. We observed participants during each trial and then asked questions about their experienceparticularly comfort, nausea, ease of use, and overall preference. Through this preliminary 
technique: rotation delay, rotation speed, and angle threshold.

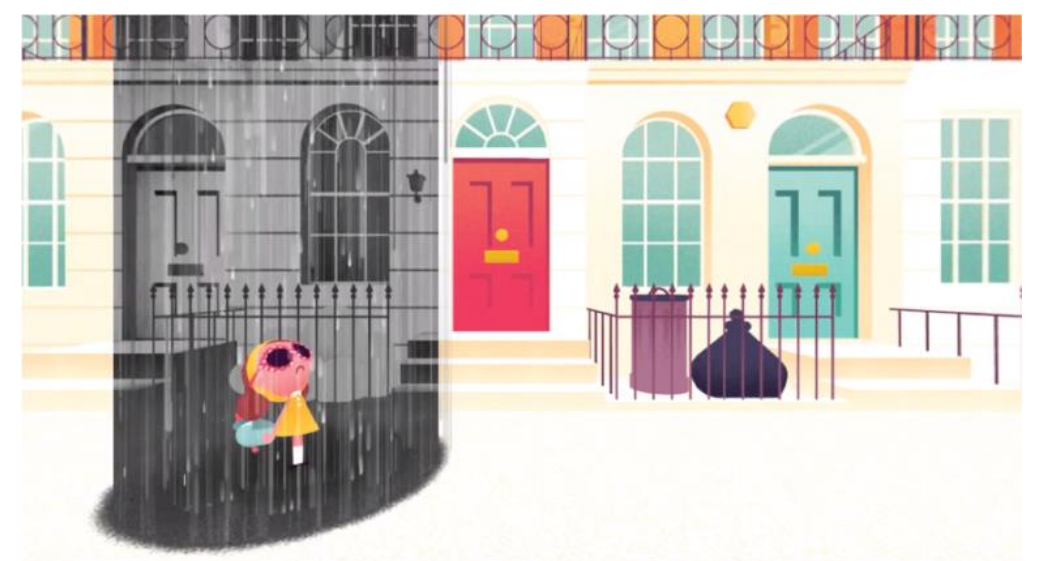

Figure 4: Still Frame from 360 Google Spotlight Story: Rain or Shine (single frame used under fair use for research and demonstration purposes).

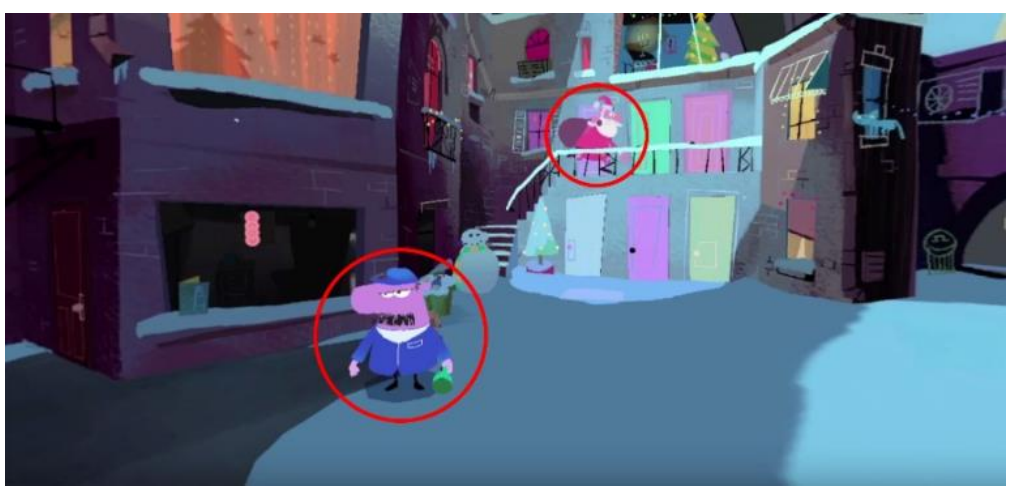

Figure 5: Still Frame from 360 Google Spotlight Story: Special Delivery (single frame used under fair use for research and demonstration purposes). The focal point of the story follows two main characters (circled in red). Since the viewer is expected to turn to follow these two characters together in 360-degree rotation, the redirection technique can assist in rotation to neutralize the need for physical turning.

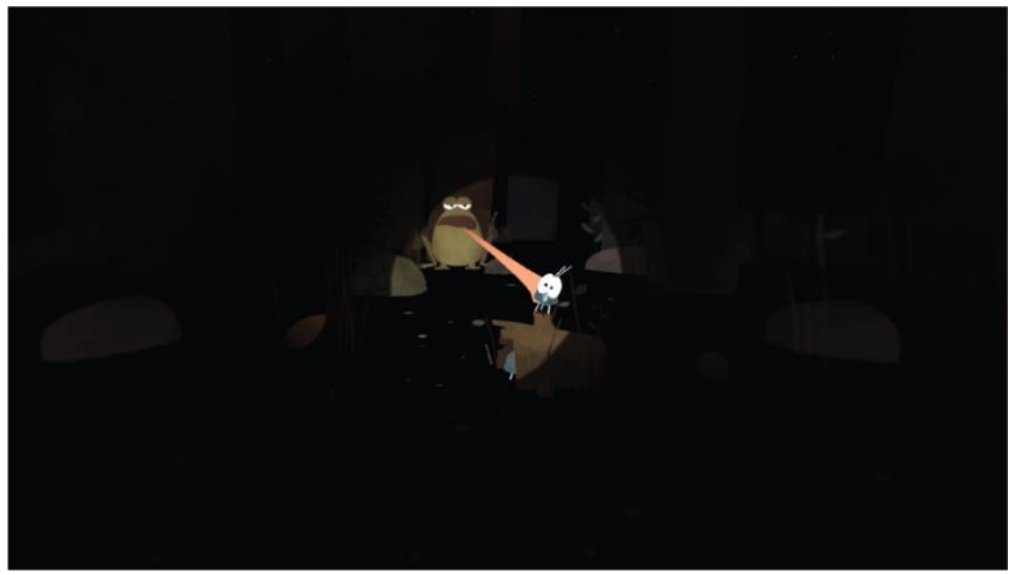

Figure 6: Still Frame from 360 Google Spotlight Story: Buggy Night (single frame used under fair use for research and demonstration purposes). 


\section{Experimental Design}

After the preliminary study, we conducted a larger formal study with 18 participants to evaluate the effects of rotation speed and rotation delay/angle threshold ratio on user experience. To minimize the number of independent variables in the experiment, we chose to combine rotation delay and angle threshold into one variable, called the delay/angle threshold. We justified this by the fact that these two variables are closely linked: the farther the user must turn his or her head to activate the scene rotation, the less time the user should have to maintain that rotation. Similarly, a lower angle threshold should require a longer delay before activating the scene rotation.

We conducted a mixed design study in which rotation speed was tested within subjects and delay/angle threshold was tested between subjects. We chose to have rotation speed tested within subjects because we believed it would be the most influential parameter, and thus it would be best for users to be able to directly compare the different rotation speeds. All subjects also viewed a video without the technique; this control trial provided a standard baseline against which users could compare the scene rotation.

We used a Latin square design to balance the order in which the techniques and videos were presented to the users. This helped to reduce any bias that the order of the videos or techniques may have on the experimental results. Appendix A lists the video and technique order used in the study.

\section{Testing Environment and Parameters}

For the formal user study, we used an HTC Vive HMD. We chose to switch from a Samsung Gear VR to the HTC Vive because the Vive allows for higher resolution videos to be played and reduces the likelihood of the video stuttering, which could be distracting to the user 
and affect the results of the study. Additionally, by using the Vive, the experimenter was able to observe what the user was looking at throughout the study.

We used the same 3 videos for the formal user study as we did for the pilot study. We tested two parameter values for rotation speed and two parameter values for the delay/angle threshold. The slow rotation parameter value used an average speed of 3 degrees per second, and the fast rotation parameter value used an average speed of 13 degrees per second. The first delay/angle threshold preset had a delay of 2 seconds and an angle threshold of 45 degrees, and the second delay/angle threshold preset had a delay of 4 seconds and an angle threshold of 25 degrees. We derived these values from the results and feedback from our preliminary evaluation.

During the study, participants sat in a non-rotating chair to watch all of the videos. We chose to use a non-rotating chair to replicate the situation where our technique would be most useful: situations in which the user is not able to freely move about or rotate their body.

\section{Procedure}

The study involved participants completing a series of questionnaires and watching a series of 360 videos in VR. At the beginning of the study, participants were given an informed consent form to read and sign. Then, each participant filled out a background questionnaire, which included questions about the participant's age, gender, occupation, major and degree program (if applicable), computer usage and experience, and experience with VR.

The experimenter then explained that the user would be watching a series of 3 VR videos and providing feedback about them. Participants were not told about the scene rotation technique being tested. We chose to do this because we desired to discover if participants notice the scene rotation at all, and disclosing the technique to the participants might bias them to notice the rotation. For each trial, the participant sat in a non-rotating chair and was instructed to put on the 
HMD and adjust the straps and lenses until he or she felt comfortable. Before watching any of the videos, participants viewed a sample 3D environment in VR using the HMD. Each participant was given as long as he or she desired to view the environment and to get used to using an HMD and viewing a scene in VR. We provided this opportunity to reduce any bias that may occur from participants who have never used VR before, and thus may take some time to acclimate to VR. Next, participants watched the three 360 videos in the order and with the corresponding techniques based on the Latin square design. After each video, the participants completed a Simulator Sickness \& Comfort Questionnaire, which asked them to rate various symptoms related to simulator sickness. The experimenter then conducted a short informal interview with the following questions:

1. How as the experience?

2. Did you notice anything interesting about the experience?

3. Did you feel distracted at all during the experience?

4. Did you feel nauseous during the experience?

The questions were intentionally vague to avoid informing the user about the scene rotation technique if they did not notice it themselves. After watching all three videos, participants completed an experience survey, which asked them to rate various aspects of each of the videos they watched. Finally, another informal interview was conducted in which the following questions were asked of the participants:

1. Were you able to follow the focal points easily during the videos?

2. Did you notice anything strange with how the videos moved? 
At this point, the experimenter explained to the participants the technique being tested and gathered feedback on how participants felt about the different rotation speeds and about the scene rotation compared to no rotation at all.

\section{Participants}

The experiment consisted of 18 participants, 9 of whom experienced the first delay/angle threshold ratio preset and 9 of whom viewed the second preset. There were 11 female and 7 male participants. Participants' ages ranged from 18-46, with a median age of 21 . Fifteen participants were undergraduate students, two were graduate students, and one was not a student. All participants were well-experienced with computers, with a median self-reported weekly computer usage of 50 hours and a mean of 49 hours. All participants reported having at least some experience with 360 videos, whether in virtual reality or simply viewed on a phone or tablet. When asked to rate their experience with virtual reality on a scale from 1 to 10 , five participants rated themselves 1-3, eight participants rated themselves 4-7, and five participants rated themselves 8-10. 


\section{CHAPTER V}

\section{RESULTS}

To analyze the results of our study, we will look at both the quantitative results drawn from the experience surveys and recorded rotation data and the qualitative results drawn from observations and the interviews.

\section{Quantitative Results}

\section{Experience Surveys}

When analyzing the results of the experience surveys, we found only one instance where there was a significant difference between the first and second delay/angle threshold preset groups, which was present in the question "I felt tired while watching the video." However, the biggest difference in responses to this question occurred for the control speed, which ideally should be similar, since the control speeds did not have a delay/angle threshold. As such, there does not appear to be a significant difference between the two delay/angle threshold groups in the experience survey results, so for the rest of the discussion of these results, we will consider both delay/angle threshold groups together and analyze any differences in the speed parameter.

To analyze any significant differences for the speed parameter values, we looked for significant differences between the slow speed and the control, between the fast speed and the control, and between the slow speed and the fast speed. We found several areas where there are significant differences between the speed parameter values. First, the question "I felt distracted during the experience" showed significant differences when comparing the slow and fast speeds, 
which shows there is a significant difference between the results. Figure 7 shows a box-andwhisker plot for the results from this question.

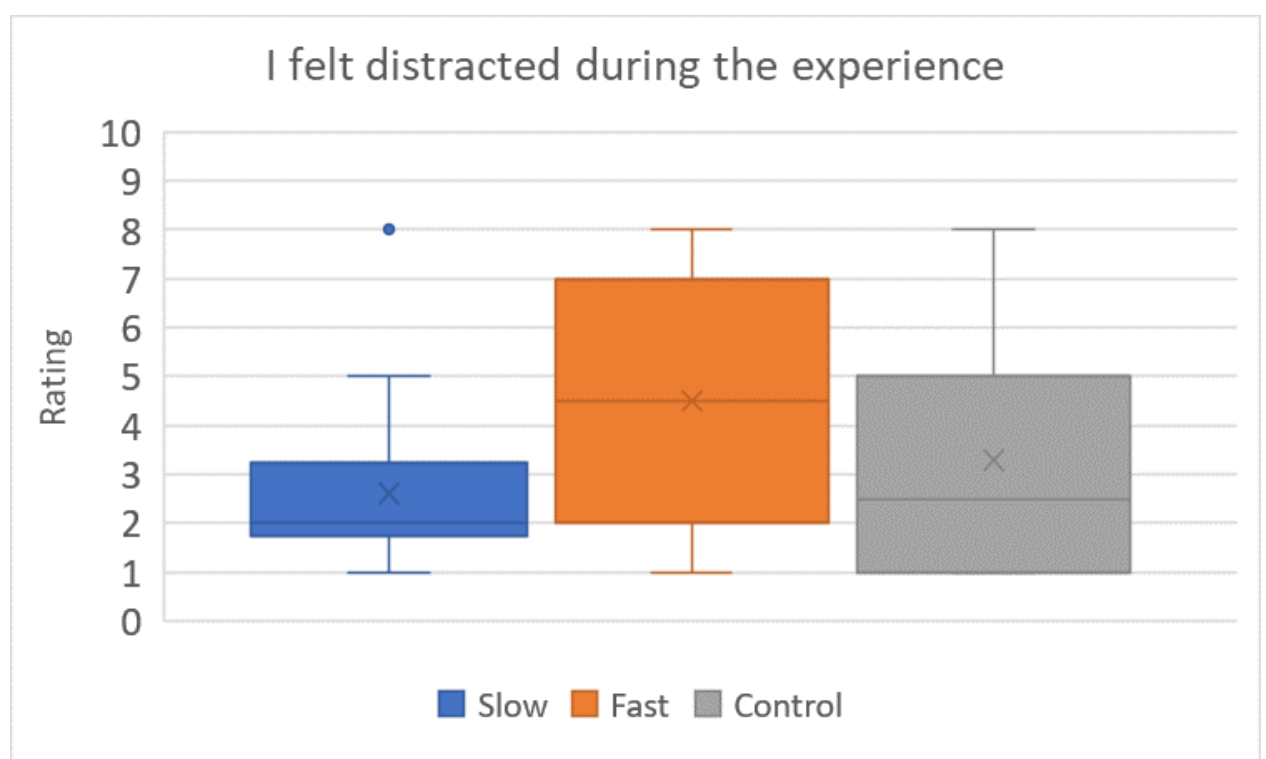

Figure 7: Ratings for the question "I felt distracted during the experience" for each speed parameter value. Higher values correspond to worse experiences.

Next, the question "The overall experience of watching the video was comfortable" also showed another significant difference in user opinion between the speed presets. Figure 8 shows a boxand-whisker plot for the results from this question. The question "The virtual reality experience was similar to the real world" also showed significant differences when comparing the fast speeed and control and when comparing the fast and slow speeds; however, there were several instances of participants not understanding exactly what the question was asking, and it is possible participants misinterpreted the question by considering the animated nature of the videos rather than the naturalness of the camera movements. Thus, the results of this difference are unclear. Next, we found significant results in the question "I felt symptoms of dizzziness while watching the video" when comparing the fast rotation speed and the control and when comparing the fast and slow rotation speeds; thus, the fast rotation speed proved significantly 
worse in this aspect than both the control and the slow rotation speed. Figure 9 shows the boxand-whisker plot for this question.

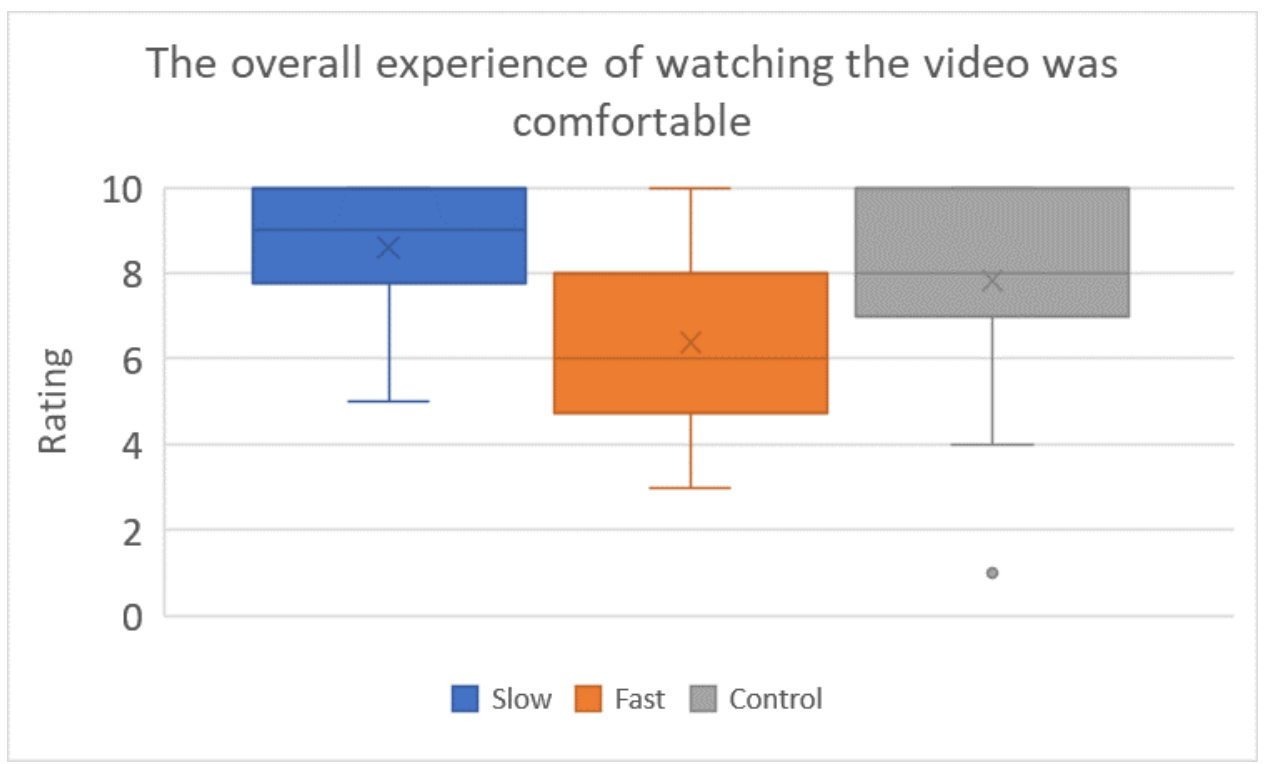

Figure 8: Ratings for the question "The overall experience of watching the video was comfortable" for each speed parameter value. Higher values correspond to better experiences.

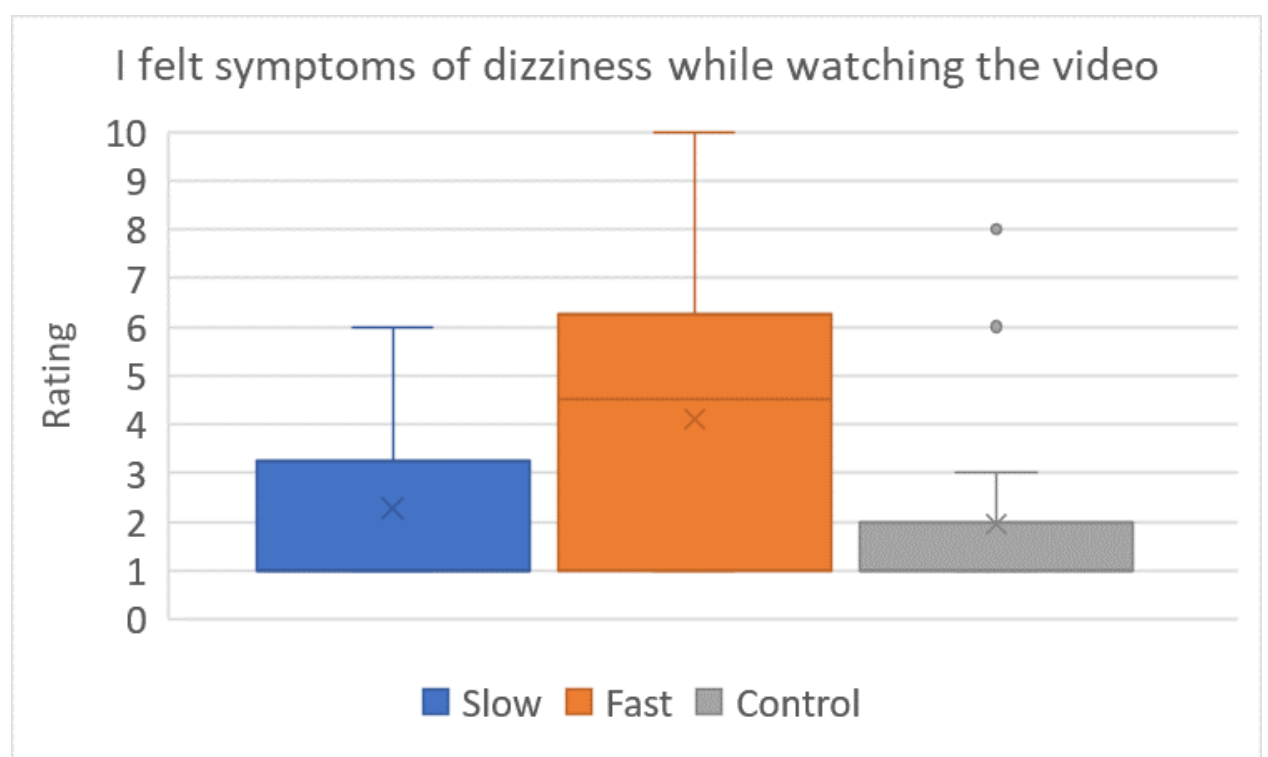

Figure 9: Ratings for the question "I felt symptoms of dizziness while watching the video" for each speed parameter value. Higher values correspond to worse experiences.

Lastly, the question "Please rate how interested you would be in using each version for home entertainment" demonstrates a significant difference when comparing the fast speed and the 
control and when comparing the slow and fast speeds, which demonstrates a significant preference for both the control and the slow speed over the fast speed. Figure 10 shows the results for this question.

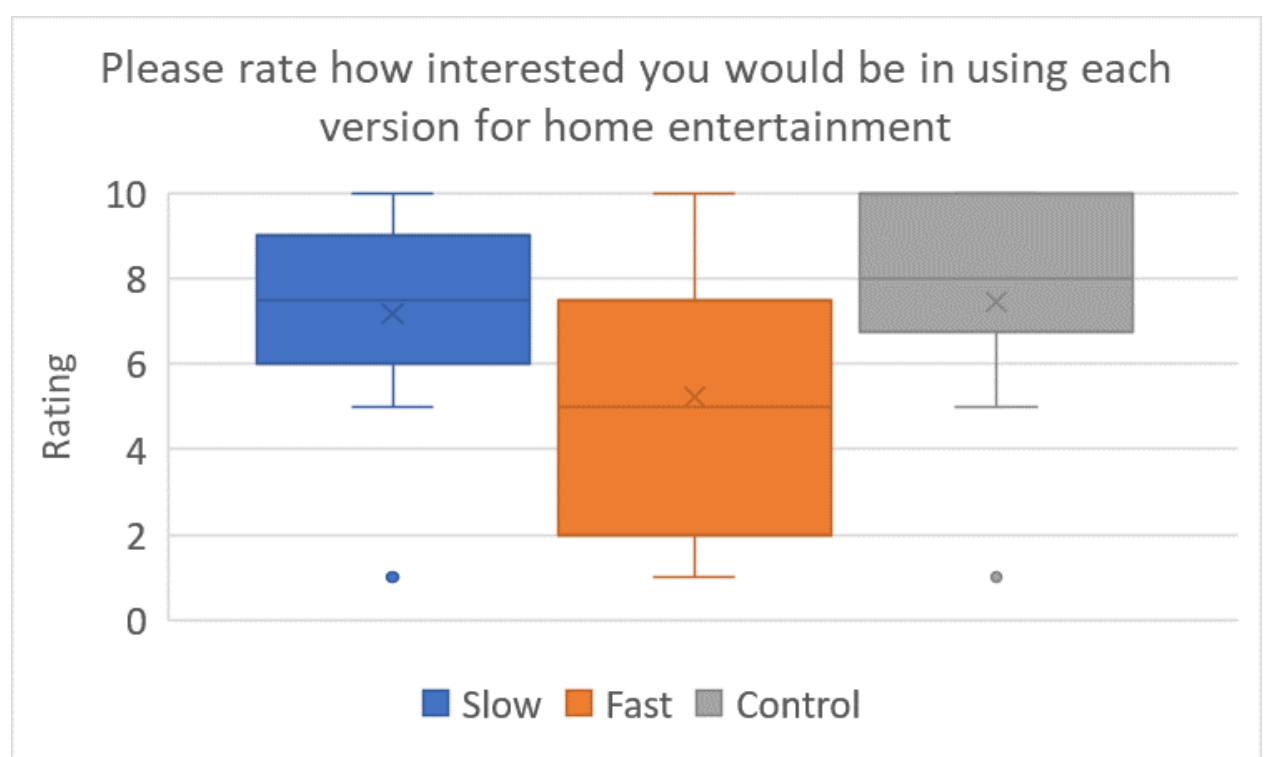

Figure 10: Ratings for the question "Please rate how interested you would be in using each version for home entertainment" for each speed parameter value. Higher values correspond to better experiences.

Overall the responses from the user experience surveys show significant results that demonstrate that the fast rotation speed is less desirable than both the slow rotation speed and the control. Interestingly, there were no significant differences between ratings for the slow rotation speed and ratings for the control, providing evidence that the slow rotation speed does not have a significant influence on user experience.

\section{Rotation Data}

Throughout the user studies, our technique implementation recorded the user's head rotation over time and output the data to a file for analysis. We are primarily concerned with the y-rotation (or yaw rotation) of the user's head, as this is the rotation affected by the scene rotation. While analysis of this data does not give direct results about the user's preferences or enjoyment of the experience, it can provide insights into how the scene rotation may affect these attributes. 
Each combination of video, rotation speed, and delay/angle threshold occurred three times throughout the user studies. These three trials were averaged to create an average head rotation over time for each combination of parameters. Appendix B lists the graphs of head rotation over time for each combination.

Upon visual analysis of the graphs of head rotation over time, a few interesting trends appear. First, there do not appear to be many significant differences in the graphs between delay/angle thresholds (i.e., delay/angle threshold does not appear to have a significant effect on the user's head rotation over time across videos or speeds). This is consistent with the data collected from the experience surveys, which showed minimal influence of delay/angle threshold on user experience.

As expected, the scene-rotation technique did have a measurable effect on head rotation with both speeds. The graphs clearly show that the trials using the rotation technique had, in general, fewer extreme angles and extreme angles with shorter durations than the control trials. This demonstrates that the scene rotation technique is, in fact, accomplishing its purpose of preventing the user from needing to maintain an extreme head angle for an extended duration of time; the graphs representing the scene rotation trials tend to drift back toward 0 degrees after they have reached an extreme angle. The primary exception to this trend occurs with the graphs for the Buggy Night video. This can likely be explained by the content of the video. Buggy Night contains a focal point which moves about the scene quickly compared to the other videos; as a result, users rarely maintained their head at an extreme angle for enough time to activate the scene rotation technique. Thus, the graphs of head rotation over time for Buggy Night appear mostly similar across all rotation speeds and delay/angle thresholds. 
One limitation with our measurement of user head rotation over time is that we failed to record during which time periods the scene rotation technique was active. Therefore, it is difficult to decipher from the raw data when the user rotated his or her head due to the scene rotation technique and when the user rotated his or her head without the technique, simply to look around the scene. Certain graph features can give clues as to the user's behavior. For example, a line slowly moving from an extreme angle toward 0 degrees is an indicator that the user was following the scene rotation, and a quick spike in the graph may indicate the user simply turned their head to a specific direction to follow an action; however, without data on the time periods when the scene rotation technique was active, it is difficult to know for sure. Thus, any future studies would benefit from recording the times when the scene rotation technique is active in addition to user head rotation data.

\section{Qualitative Results}

User responses during the informal interviews provided valuable insight into users' preferences for the rotation technique. First, to measure whether a user noticed the scene rotation, we analyzed the responses to the informal interviews that took place after each trial. If the user commented on any type of involuntary head rotation, scene rotation, or similar effect, it was marked as the user having noticed the scene rotation. Tables 1 and 2 list the results of whether users noticed the scene rotation, broken down by video, rotation speed, and delay/angle threshold. 18 of 18 participants $(100 \%)$ noticed the scene rotation with the fast rotation speed. Thus, the chosen value for the fast rotation speed appears too fast to go unnoticed by users. Contrarily, only $33.33 \%$ of participants with delay/angle threshold 1 noticed the slow rotation, and $44.44 \%$ of participants with delay/angle threshold 2 noticed the slow rotation. Interestingly, the video seemed to influence if the participant noticed the slow rotation. Specifically, rotation was noticed most in 
Special Delivery compared to Rain or Shine and Buggy Night. This can likely be explained by the video content. Rain or Shine featured a main character that moved 360 degrees throughout the scene, and the camera panned and moved about the scene throughout the video, as well. In Special Delivery, most of the action took place within a 180-degree field of view, and only brief vertical camera movement occurred. Thus, it is likely that the character and camera movement in Rain or Shine helped to mask the scene rotation that occurred. In Buggy Night, as stated previously, the focal point moved around the scene quickly, resulting in fewer instances of the scene rotation occurring. Also, the backdrop for Buggy Night was much darker than those in other videos, which may have also contributed to fewer participants noticing the scene rotation in this video.

Table 1: Noticeability of scene rotation for delay/angle threshold 1

\begin{tabular}{|c|c|c|c|c|}
\hline & Noticed & Didn't Notice & $\%$ Noticed & \% Noticed \\
\hline Rain or Shine, Slow & 0 & 3 & 0 & \multirow[t]{3}{*}{33.33} \\
\hline Special Delivery, Slow & 3 & 0 & 100 & \\
\hline Buggy Night, Slow & 0 & 3 & 0 & \\
\hline Rain or Shine, Fast & 3 & 0 & 100 & \multirow[t]{3}{*}{100} \\
\hline Special Delivery, Fast & 3 & 0 & 100 & \\
\hline Buggy Night, Fast & 3 & 0 & 100 & \\
\hline
\end{tabular}

Table 2: Noticeability of scene rotation for delay/angle threshold 2

\begin{tabular}{|c|c|c|c|c|}
\hline & Noticed & Didn't Notice & $\%$ Noticed & \% Noticed \\
\hline Rain or Shine, Slow & 1 & 2 & 33.33 & \multirow{3}{*}{44.44} \\
\hline Special Delivery, Slow & 2 & 1 & 66.67 & \\
\hline Buggy Night, Slow & 1 & 2 & 33.33 & \\
\hline Rain or Shine, Fast & 3 & 0 & 100 & \multirow[t]{3}{*}{100} \\
\hline Special Delivery, Fast & 3 & 0 & 100 & \\
\hline Buggy Night, Fast & 3 & 0 & 100 & \\
\hline
\end{tabular}

During the final informal interview, after the participant completed all three trials, the experimenter asked each participant their preference for the faster or the slower rotation and for scene rotation in general or no rotation at all. Amongst the participants who used delay/angle 
threshold 1, 8 of 8 participants $(100 \%)$ who gave a preference preferred the slow rotation to the fast, and 5 of 7 participants $(71.43 \%)$ who gave a preference preferred some scene rotation to no scene rotation at all. Within the delay/angle threshold 2 group, 7 of 9 participants $(77.78 \%)$ preferred the slow rotation to the fast, and 7 of 9 participants $(77.78 \%)$ preferred some scene rotation, while 1 of $9(11.11 \%)$ preferred no scene rotation, and 1 of $9(11.11 \%)$ said that it would depend on the situation they were in. Overall, 15 of 17 participants $(88.24 \%)$ who gave a preference preferred the slow rotation to the fast, and 12 of $15(80 \%)$ who gave a preference preferred some scene rotation to no scene rotation. Some participants even stated that they felt like the scene rotation added to the experience. However, about half of the participants who preferred the rotation stated that they would only prefer it given the physical constraints present in the experiment; if they were able to move freely about the environment, they would prefer no scene rotation.

Overall, the primary complaint of participants about the fast scene rotation was that it was too jarring and made the user feel a bit dizzy, distracted, or disconnected from the experience. 7 of 9 participants $(77.78 \%)$ from delay/angle threshold 1 and 6 of 9 participants $(66.67 \%)$ from delay/angle threshold 2 complained of this. Some people (2 of 9 participants $(22.22 \%)$ from delay/angle threshold 1 and 2 of 9 participants (22.22\%) from delay/angle threshold 2) also complained that the rotation seemed confusing, as it added unnecessary movement and did not seem to make sense, as though the rotation were trying to get the user to look at something even though they were already looking at the focal point. This is consistent with some of the observations made during the trials; there were several instances in which a user would be watching the focal point, the fast scene rotation would occur, and the user would maintain their head in the same physical position. Thus, instead of following the focal point with the rotation, the 
focal point was instead rotated away from the participant, and he or she was left with a different, often unimportant, object placed in front of them. This outcome appeared to occur more often in the second delay/angle threshold preset, as the scene rotation would occur at a smaller angle threshold, when the user did not actually need any scene rotation to occur. This is the only noticeable difference found between the two delay/angle threshold presets. This suggests that the angle threshold chosen for delay/angle threshold 2 may be too small.

Users' responses to the informal interview questions gave further explanations regarding their preferences for certain speeds or techniques. The following are some representative quotes from the user studies:

"I didn't like having to move my body 360 around to follow the story." (discussing the control trial without redirection)

"The whole scene was rotating, and until I was sure that it was, it felt strange, it felt like the goggles were shifting on my face until I was aware that it was actually the scene."

"[The fast rotation was] the most disorienting, because the scene rotated around me, and sometimes that motion didn't make sense; it would bring characters to the front of my vision, and other ones would rotate when it shouldn't."

"No, the rotation was so slow that it was not distracting. I liked the pace of the rotation, it was not unnoticeable, but not in your face." (discussing the slow rotation)

"I preferred the rotation. I didn't like bending my neck."

Overall, users preferred the slower rotation to the faster rotation, and preferred at least some rotation to none given the physical conditions. Users who did not notice the slow rotation at all (6 of 9 participants (66.67\%) from delay/angle threshold 1 and 5 of 9 participants $(55.56 \%)$ from 
delay/angle threshold 2) invariably preferred the slow rotation, as it did not distract them and resulted in less need for the user to turn his or her head and body. Furthermore, beyond minor dizziness or nausea reported by a few of the participants, no technique parameter values appeared to cause serious sickness or discomfort in any of the participants (the most serious reported case of discomfort by a user was a result of the vertical camera movement in Special Delivery, which is an aspect of the video itself and not the rotation technique). 


\section{CHAPTER VI}

\section{CONCLUSION}

Our research explores the effectiveness of a scene rotation technique for redirecting a viewer's gaze in immersive movies. Our technique employs a mechanism similar to washout filters used in motion simulators; when the user rotates his or her head beyond a certain angle, the technique rotates the scene to bring what the user is looking at, and by extension the user's head, toward the user's physical forward direction. In our research, we tested the feasibility of the technique and the effectiveness of different parameter values on user experience: specifically, rotation speed and delay/angle threshold.

Our user study found that the scene rotation technique was effective at redirecting a user's gaze to his or her physical forward direction. Users overwhelmingly preferred the slow scene rotation speed to the fast scene rotation speed, and generally preferred having scene rotation to not having it, especially given the physical constraints of the experimental environment. We did not find significant differences between the two delay/angle threshold ratios tested, other than that the lower angle threshold tended to activate the scene rotation at times when it was not necessary. The results of the user studies are promising for future use of the technique, especially for our intended use case of situations where a user is not able to physically turn or move about an environment.

The primary contribution of our research is the insight and improved understanding that it provides regarding how users respond to the redirected scene rotation technique. The results demonstrate that the technique is likely feasible for use in immersive movies, as users reported positive experiences with the technique, especially with the slow rotation speed. Further research should be conducted to better understand how an optimal scene rotation speed can be achieved. 
For example, perhaps a slower rotation speed would reduce noticeability while still effectively redirecting the user's gaze to the physical forward direction, or the scene rotation speed could be directly mapped to the movement speed of the focal point in the video. Additionally, studies that employ longer immersive movies, and perhaps live-action movies, as well, would be useful to demonstrate the effectiveness and robustness of the technique across different video styles and durations. It would also be interesting to explore the possibility of implementing a dynamic delay/angle threshold ratio that responds to the user's head rotation. Another potential area for further research involves altering the technique so that it only rotates when the user is looking at the main focal point of the video; this would require manually coding the location of the focal point of the video or using a computer vision algorithm to analyze and determine the location of the focal point. This could prove interesting when considering how the technique could be used from a directorial perspective. 


\section{REFERENCES}

[1] J. Becker and T. Ngo. Mitigating visually-induced motion sickness in virtual reality. 2016.

[2] B. Bolte and M. Lappe. Subliminal reorientation and repositioning in immersive virtual environments using saccadic suppression. IEEE transactions on visualization and computer graphics, 21(4):545-552, 2015.

[3] D. A. Bowman, R. P. McMahan, and E. D. Ragan. Questioning naturalism in 3D user interfaces. Communications of the ACM, 55(9):78-88, 2012.

[4] S. H. Chen and L. C. Fu. An optimal washout filter design for a motion platform with senseless and angular scaling maneuvers. In Proceedings of the 2010 American Control Conference, pp. 4295-4300, June 2010. doi: 10.1109/ACC.2010.5530820

[5] S. Razzaque, Z. Kohn, and M. C. Whitton. Redirected walking. In Proceedings of EUROGRAPHICS, vol. 9, pp. 105-106. Manchester, UK, 2001.

[6] S. P. Sargunam, K. R. Moghadam, M. Suhail, and E. D. Ragan. Guided head rotation and amplified head rotation: Evaluating semi-natural travel and viewing techniques in virtual reality. In 2017 IEEE Virtual Reality (VR), pp. 19-28, March 2017. doi:

0.1109/VR.2017.7892227

[7] P. Schmitz, J. Hildebrandt, A. C. Valdez, L. Kobbelt and M. Ziefle, "You Spin my Head Right Round: Threshold of Limited Immersion for Rotation Gains in Redirected Walking," in IEEE Transactions on Visualization and Computer Graphics, vol. 24, no. 4, pp. 16231632, April 2018. doi: 10.1109/TVCG.2018.2793671

[8] T. Stebbins and E. D. Ragan. "Redirected Scene Rotation for Immersive Movie Experiences," 2018 IEEE Virtual Reality(VR), Reutlingen, Germany, 2018.

[9] E. A. Suma, G. Bruder, F. Steinicke, D. M. Krum, and M. Bolas. A taxonomy for deploying redirection techniques in immersive virtual environments. In 2012 IEEE Virtual Reality Workshops (VRW), pp. 43-46, March 2012. doi: 10.1109/VR.2012.6180877 


\section{APPENDIX A}

The tables below list the video and technique orders that were used in the Latin square design for our study.

Table 3: Delay/Angle Threshold 1

\begin{tabular}{|l|l|l|l|l|l|l|}
\hline $\begin{array}{l}\text { Participant } \\
\text { ID }\end{array}$ & $\begin{array}{l}\text { Session 1 } \\
\text { Video }\end{array}$ & $\begin{array}{l}\text { Session 1 } \\
\text { Speed }\end{array}$ & $\begin{array}{l}\text { Session 2 } \\
\text { Video }\end{array}$ & $\begin{array}{l}\text { Session 2 } \\
\text { Speed }\end{array}$ & $\begin{array}{l}\text { Session 3 } \\
\text { Video }\end{array}$ & $\begin{array}{l}\text { Session 3 } \\
\text { Speed }\end{array}$ \\
\hline 1 & 1 & 1 & 2 & 2 & 3 & 3 \\
\hline 2 & 2 & 1 & 3 & 2 & 1 & 3 \\
\hline 3 & 3 & 1 & 1 & 2 & 2 & 3 \\
\hline 4 & 1 & 2 & 2 & 3 & 3 & 1 \\
\hline 5 & 2 & 2 & 3 & 3 & 1 & 1 \\
\hline 6 & 3 & 2 & 1 & 3 & 2 & 1 \\
\hline 7 & 1 & 3 & 2 & 1 & 3 & 2 \\
\hline 8 & 2 & 3 & 3 & 1 & 1 & 2 \\
\hline 9 & 3 & 3 & 1 & 1 & 2 & 2 \\
\hline
\end{tabular}

Note: Video 1 = Rain or Shine, Video $2=$ Special Delivery, Video $3=$ Buggy Night; Speed 1= Slow, Speed 2 = Fast , Speed $3=$ Control (no rotation)

Table 4: Delay/Angle Threshold 2

\begin{tabular}{|l|l|l|l|l|l|l|}
\hline $\begin{array}{l}\text { Participant } \\
\text { ID }\end{array}$ & $\begin{array}{l}\text { Session 1 } \\
\text { Video }\end{array}$ & $\begin{array}{l}\text { Session 1 } \\
\text { Speed }\end{array}$ & $\begin{array}{l}\text { Session 2 } \\
\text { Video }\end{array}$ & $\begin{array}{l}\text { Session 2 } \\
\text { Speed }\end{array}$ & $\begin{array}{l}\text { Session 3 } \\
\text { Video }\end{array}$ & $\begin{array}{l}\text { Session 3 } \\
\text { Speed }\end{array}$ \\
\hline 10 & 1 & 1 & 2 & 2 & 3 & 3 \\
\hline 11 & 2 & 1 & 3 & 2 & 1 & 3 \\
\hline 12 & 3 & 1 & 1 & 2 & 2 & 3 \\
\hline 13 & 1 & 2 & 2 & 3 & 3 & 1 \\
\hline 14 & 2 & 2 & 3 & 3 & 1 & 1 \\
\hline 15 & 3 & 2 & 1 & 3 & 2 & 1 \\
\hline 16 & 1 & 3 & 2 & 1 & 3 & 2 \\
\hline 17 & 2 & 3 & 3 & 1 & 1 & 2 \\
\hline 18 & 3 & 3 & 1 & 1 & 2 & 2 \\
\hline
\end{tabular}

Note: Video 1 = Rain or Shine, Video $2=$ Special Delivery, Video $3=$ Buggy Night ; Speed 1= Slow, Speed 2 = Fast , Speed $3=$ Control (no rotation) 


\section{APPENDIX B}

The graphs below display average user head rotation over time for each combination of video, rotation speed, and delay/angle threshold. Y rotation values of 0 degrees correspond to the user's physical forward direction and values of -180 and +180 degrees correspond to 180 degrees to the left and to the right, respectively.

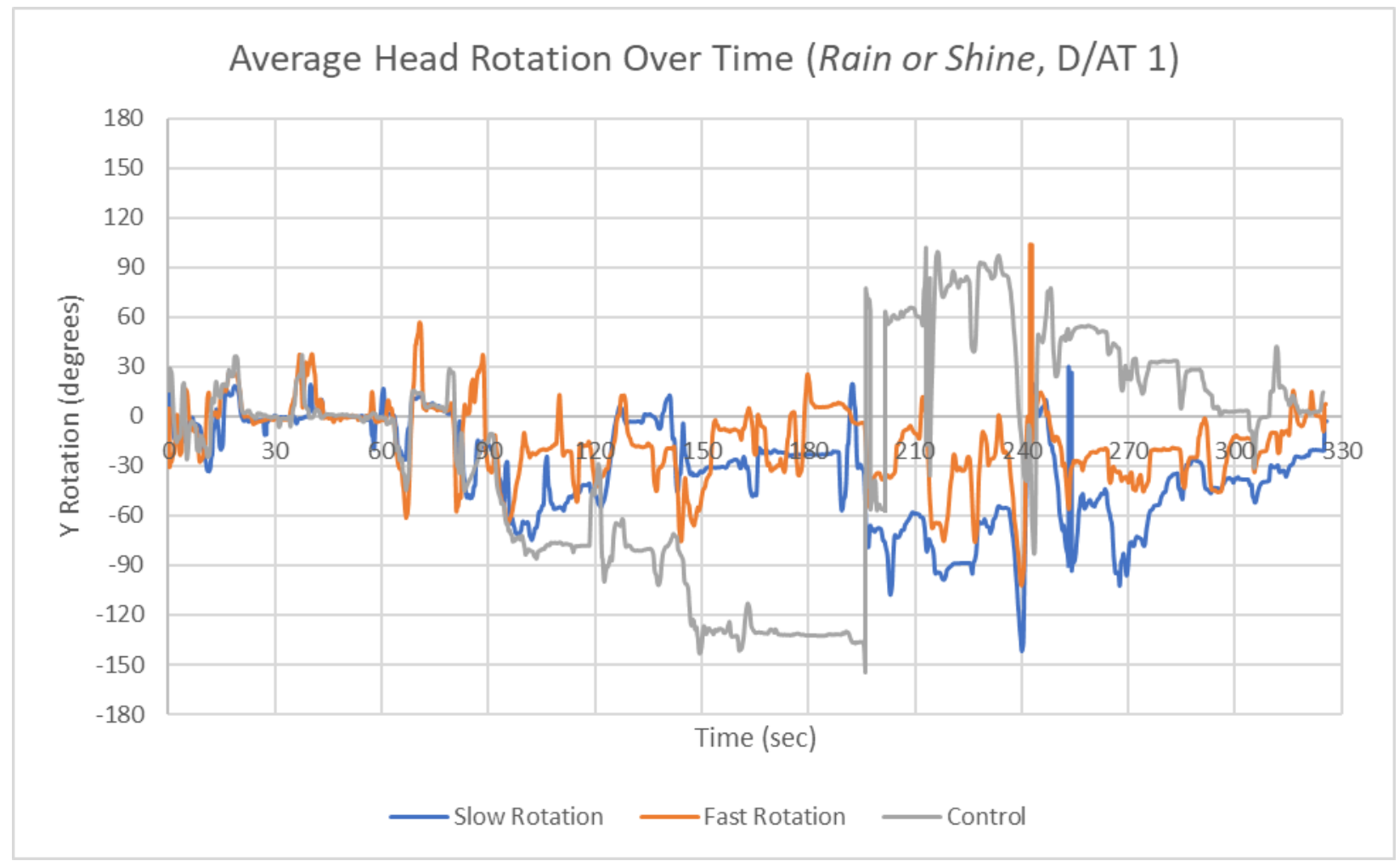

Figure 11: Graph of head rotation over time for each speed parameter value with delay/angle threshold 1 in Rain or Shine. 


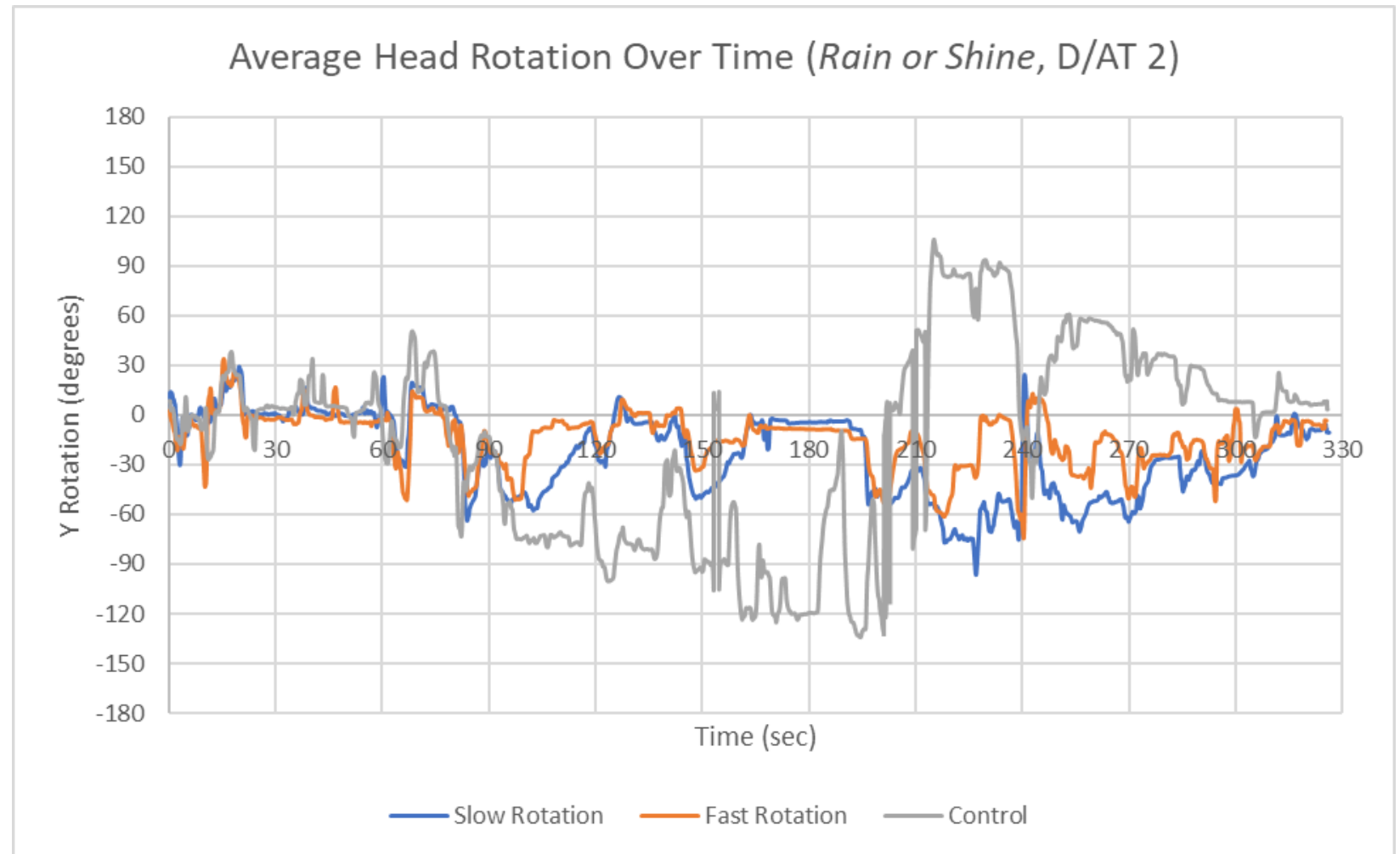

Figure 12: Graph of average head rotation over time for each speed parameter value with delay/angle threshold 2 in Rain or Shine.

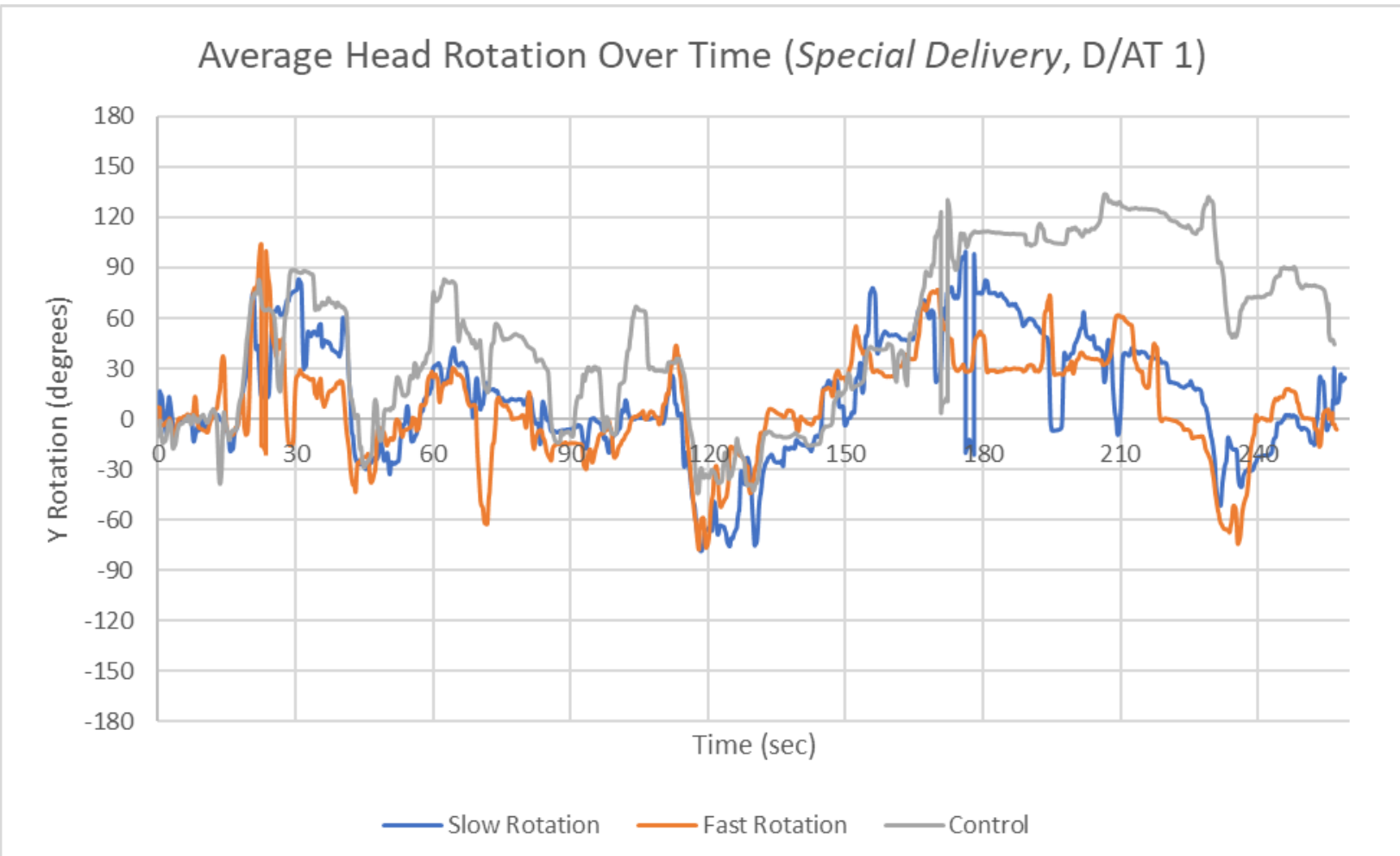

Figure 13: Graph of average head rotation over time for each speed parameter value with delay/angle threshold 1 in Special Delivery. 


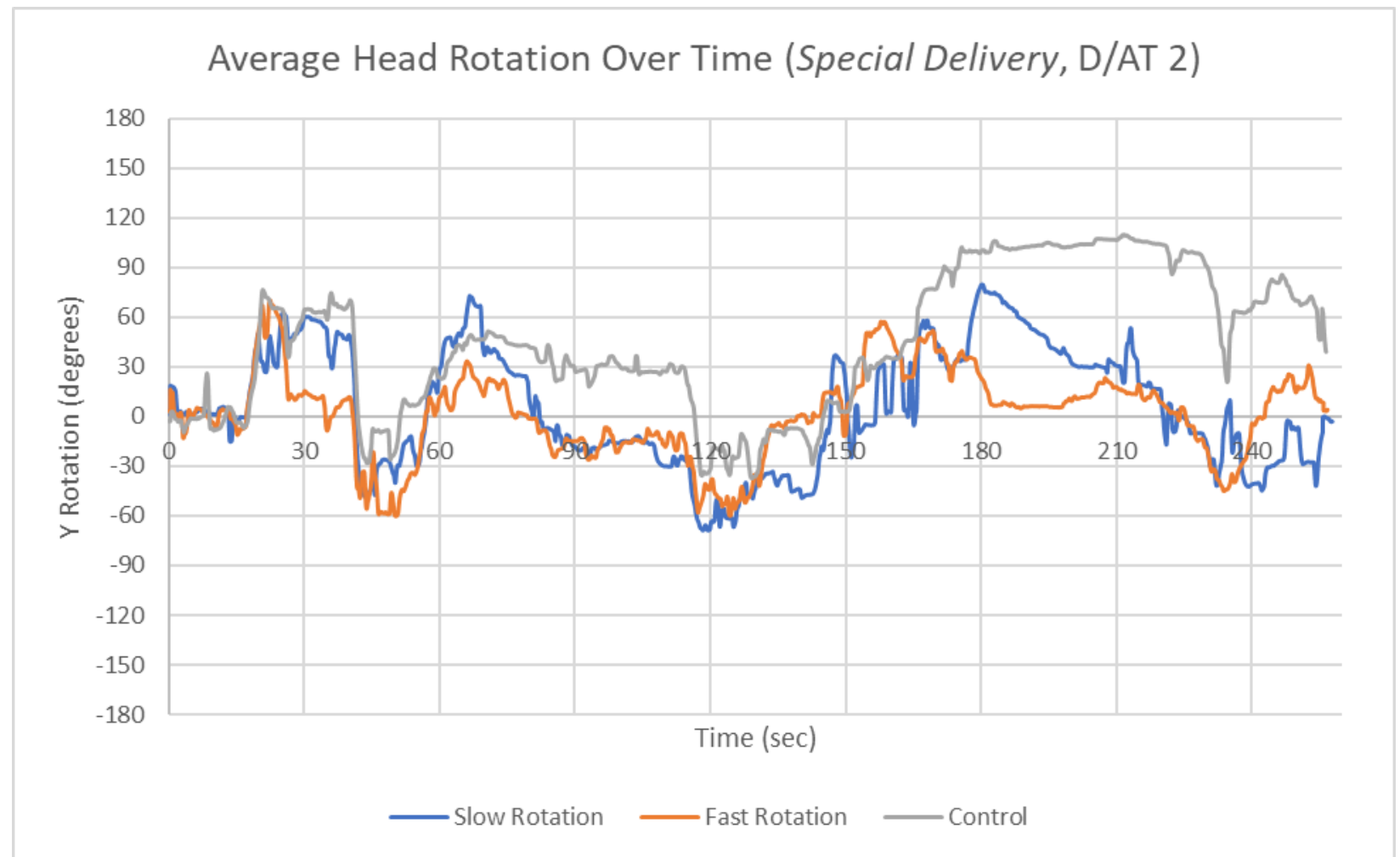

Figure 14: Graph of average head rotation over time for each speed parameter value with delay/angle threshold 2 in Special Delivery.

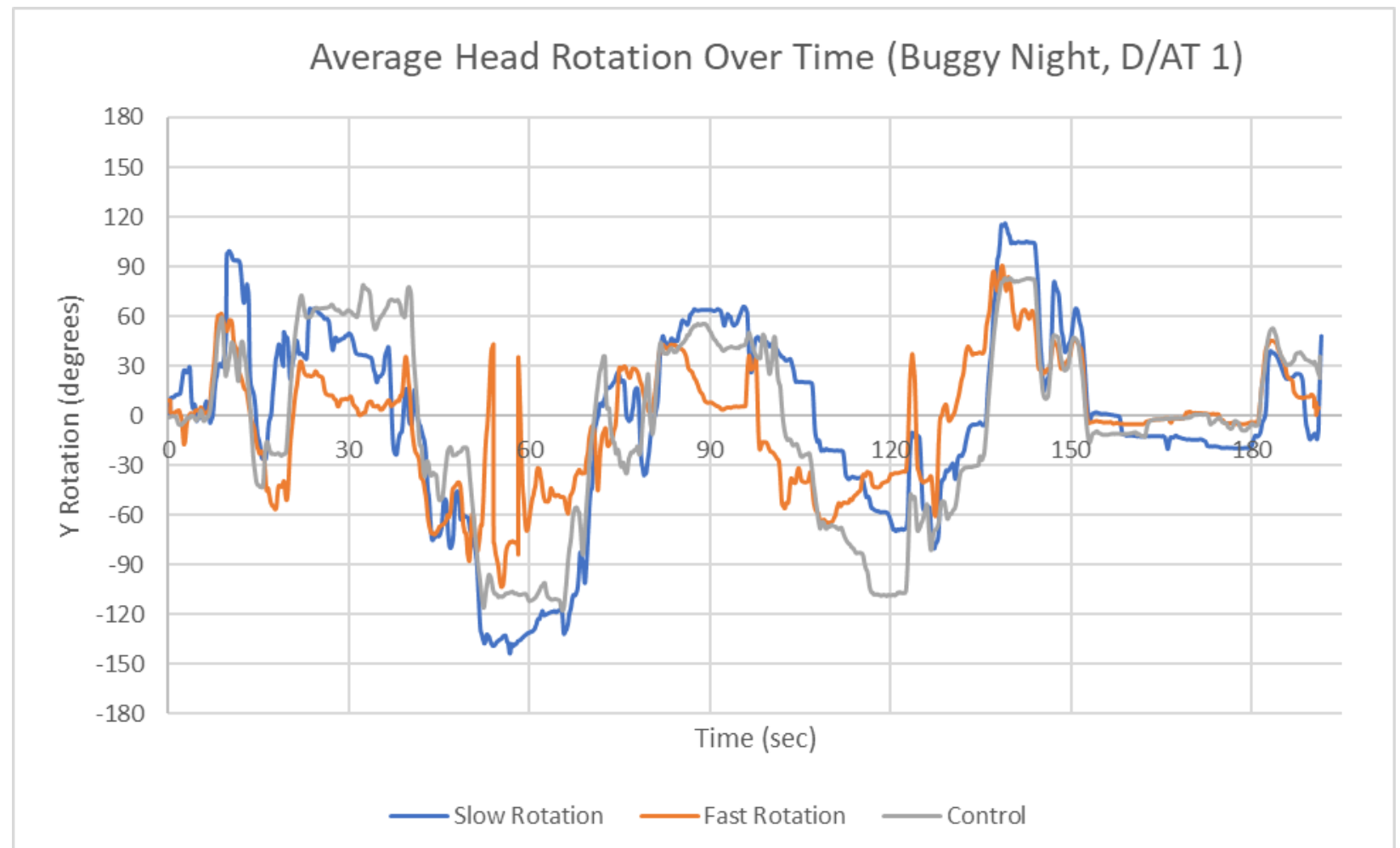

Figure 15: Graph of average head rotation over time for each speed parameter value with delay/angle threshold 1 in Buggy Night. 


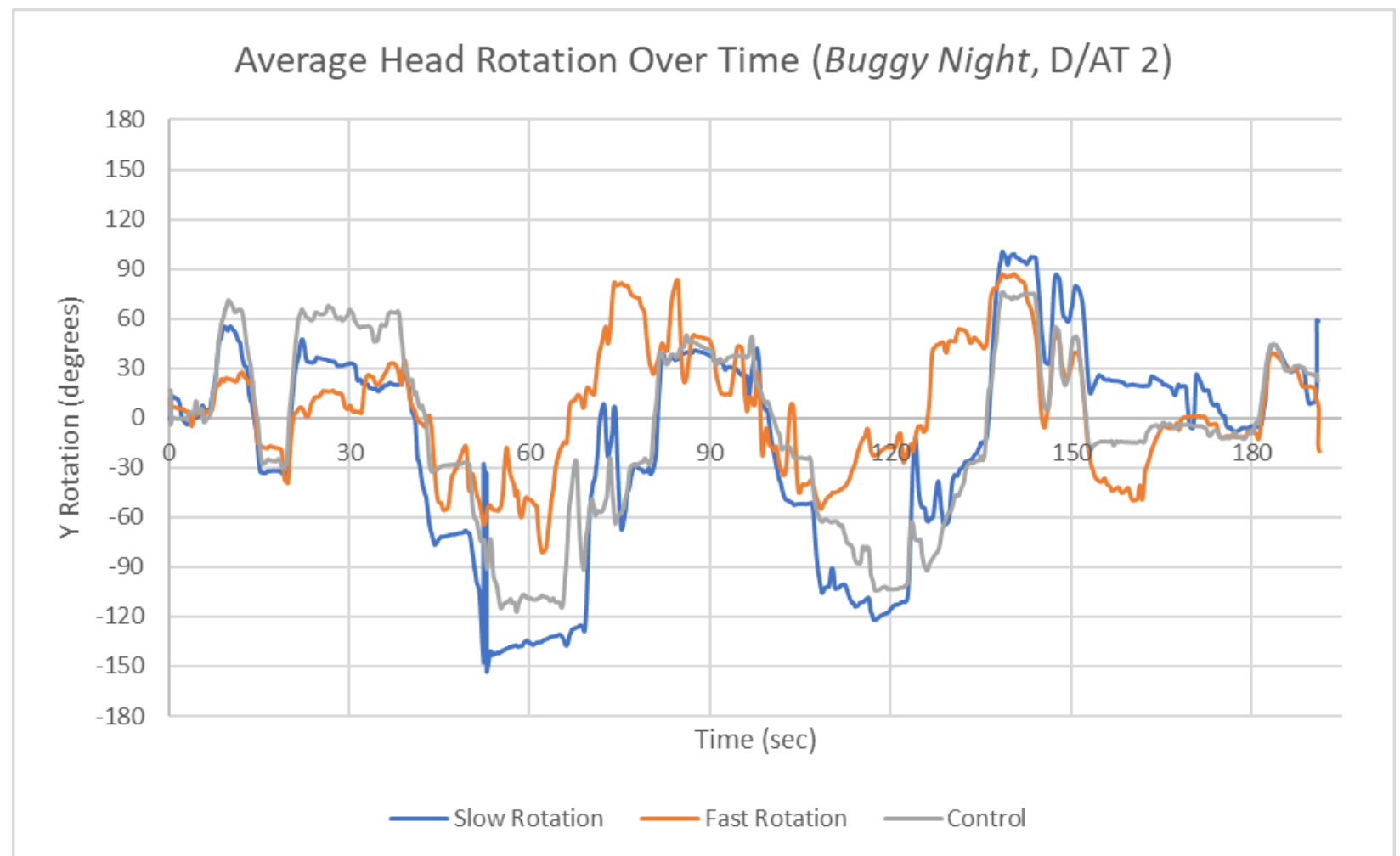

Figure 16: Graph of average head rotation over time for each speed parameter value with delay/angle threshold 2 in Buggy Night. 